\title{
Effect of p-Doped Overlayer Thickness on RF-Dispersion in GaN Junction FETs
}

\author{
A. Jiménez, D. Buttari, D. Jena, R. Coffie, S. Heikman, N. Q. Zhang, L. Shen, E. Calleja, E. Muñoz, J. Speck, \\ and U. K. Mishra, Fellow, IEEE
}

\begin{abstract}
Successive reactive ion etchings (RIE) were performed on the access regions of $p^{+}-n$ GaN JFETs. A decrease in the n-layer sheet resistance, with a consequent increase in $I_{D S S}$ was detected after complete removal of the p-layer, due to a reduction in the n-layer depletion region. An increase in RF-dispersion was experienced, as a result of the progressive reduction of screening from surface-states originally provided by the overlying $p$-cap layer. No dispersion was detected before cap removal. A continuous increase in $f_{t}$ and $f_{\max }$ was detected even before complete removal of the p-layer, due to virtual gate length reduction. It is expected that an optimized p-doped overlayer will provide current slump suppression without degradation in cutoff frequency or breakdown.
\end{abstract}

Index Terms-GaN, JFET, passivation, RF-dispersion.

\section{INTRODUCTION}

$\mathbf{I}^{\mathrm{N}}$ $\mathrm{N}$ RECENT years, GaN-based heterostructures have received increased attention because of their potential for use in high-power, high-frequency devices. Despite the considerable improvement in GaN-technology and material quality, RF-dispersion is still one of the main issues hampering device progress. RF-dispersion affects device output power and device power-added efficiency (PAE) due to a reduction in saturation current and an increase in knee voltage at high frequencies and high biases. Dispersion has been related to surface, interface [1], [2], or bulk trap states [3], and recently to polarization-related charge states at the surface or barrier [4], [5]. Surface passivation using silicon nitride has been found to mitigate RF-dispersion and microwave power degradation [2]-[4]. Further understanding of these phenomena and their physical origins are required to fully exploit the potential of $\mathrm{GaN}$ devices. In this paper the effects of p-doped cap layer thickness on the dc, small-signal characteristics, and dispersion of GaN-based junction field effect transistors (JFETs) are analyzed. GaN-based JFETs using ion-implantation [6] and metal organic chemical vapor deposition (MOCVD) [7] have

Manuscript received January 17, 2002; revised March 26, 2002. This work was supported in part by the Office of Naval Research IMPACT MURI, Office of Naval Research CANE MURI, and Air Force Office of Scientific Research Programs, and the European Space Agency (ESA). The review of this letter was arranged by Editor D. Ritter.

A. Jiménez,E. Calleja, and E. Muñoz are with the Departamento de Ingeniería Electrónica de la E.T.S.I.Telecomunicación, Universidad Politecnica de Madrid, 28040 Madrid, Spain.

D. Buttari, D. Jena, R. Coffie, S. Heikman, L. Shen, and U. K. Mishra are with the Department of Electrical and Computer Engineering, University of California, Santa Barbara, CA 93106 USA.

J. Speck is with the Materials Department, University of California, Santa Barbara, CA 93106 USA.

Publisher Item Identifier S 0741-3106(02)05269-2.
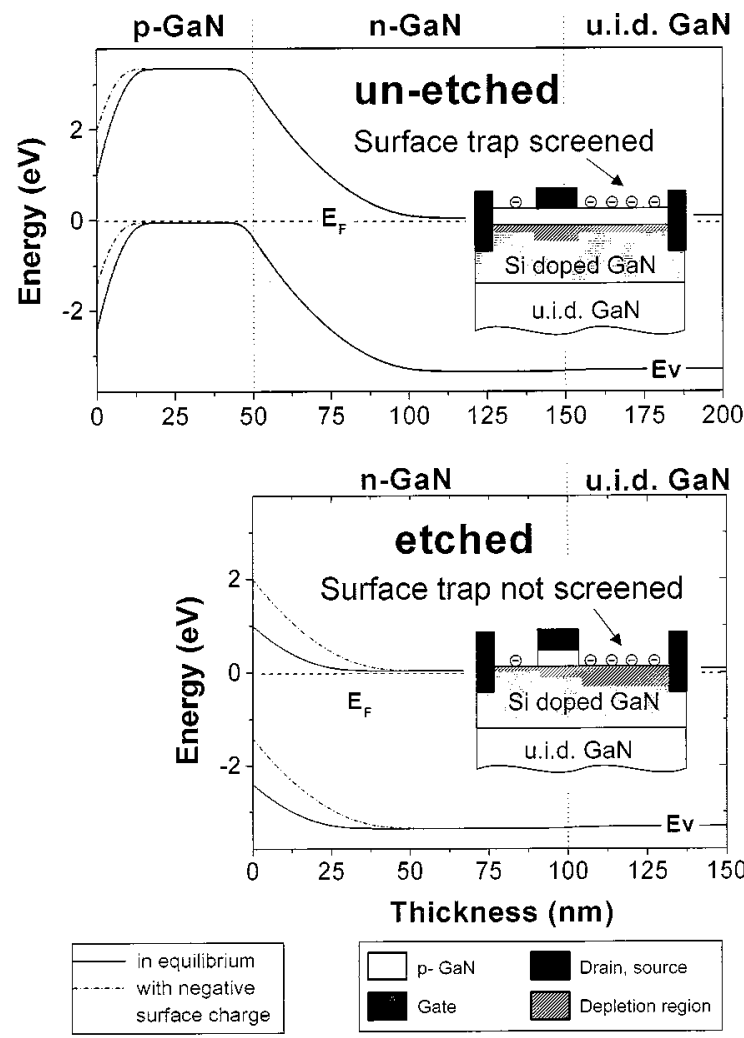

Fig. 1. Band diagram in the access regions before and after removal of the p-layer (solid line), showing its effectiveness in screening the channel from surface-states (dashed line). As a consequence of etching, the thickness of the depletion region in the n-layer decreased from 60 to $34 \mathrm{~nm}$. Schematic diagrams of the corresponding JFET structures are also shown.

been previously reported, but we report for the first time a GaN-JFET structure grown by molecular beam epitaxy (MBE). We also demonstrate for the first time that a p-doped overlayer is effective for RF dispersion suppression, with possible beneficial effects in reproducibility compared to silicon nitride passivation. Although RF performance degradation due to virtual gate extension was observed for full cap layer thickness, it is speculated that a well designed p-layer (optimized for thickness, doping, and deep-acceptor trap depth) will provide current slump suppression without affecting RF and breakdown performances.

\section{Material Growth AND DeVice Characterization}

The GaN JFET layer structure (Fig. 1) used in this study was grown by plasma-assisted molecular beam epitaxy (MBE) under Ga-stable conditions [8] on a semi-insulating MOCVD 
GaN-template grown on a $c$-plane sapphire substrate. The epitaxial structure consisted of a $1000 \AA$ A-thick undoped high-resistivity GaN buffer layer, a $1000 \AA$ Si-doped n-GaN channel, and a $500 \AA \mathrm{Mg}$-doped $\mathrm{p}^{+}-\mathrm{GaN}$ contact layer. Nominal doping concentrations were $1 \times 10^{19} \mathrm{~cm}^{-3}$ and $1 \times 10^{18} \mathrm{~cm}^{-3}$ for $\mathrm{Mg}$ and $\mathrm{Si}$, respectively.

JFETs were fabricated by $\mathrm{Ti} / \mathrm{Al} / \mathrm{Ni} / \mathrm{Au}$ ohmic contact formation, mesa isolation, $\mathrm{Pd} / \mathrm{Au} / \mathrm{Ni}$ gate definition, and reactive ion etching (RIE) of the access regions. All layers were defined by $i$-line stepper lithography.

After ohmic lithography but before metal evaporation, a selfaligned reactive ion etch (RIE) was performed in order to expose the $\mathrm{n}$-doped regions. Medium power $(60 \mathrm{~W}$ source power) etching with an etch rate of $\sim 6 \AA / s$, was applied for $105 \mathrm{~s}$ in order to remove $\sim 600 \AA$ from the surface layer.

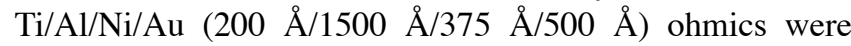
deposited by e-beam and annealed at $870{ }^{\circ} \mathrm{C}$ for $30 \mathrm{~s}$ in $\mathrm{N}_{2}$ atmosphere. Mesa isolation was performed by RIE (100 W source power) at an etch rate of about $10 \AA / \mathrm{s}$ for $300 \mathrm{~s}$, corresponding to an etch depth of about $3000 \AA$ A. In order to prevent gate metal discontinuities due to poor mesa-edge step coverage, the trench regions were filled with $2000 \AA$ of e-beam deposited silicon dioxide. Next, a Pd/Au/Ni gate $(200 \AA \AA 2000$ $\AA / 500 \AA$ ) was e-beam evaporated and annealed at $600{ }^{\circ} \mathrm{C}$ for $60 \mathrm{~s}$ in $\mathrm{N}_{2}$ gas. The Ni layer was used as an etch mask during the selective removal of the overlying p-layer in the access regions. The removal was obtained by blanket chlorine RIE etches performed at low power ( $15 \mathrm{~W}$ source power) in order to minimize ion-induced surface damage. Three different etch depths $(200,400$, and $600 \AA)$ were cumulatively reached and the results were compared to un-etched devices. Each etch was preceded by $20 \mathrm{~s} \mathrm{HCl} \operatorname{dip}\left(\mathrm{HCl}: \mathrm{H}_{2} \mathrm{O} 1: 10\right)$. Finally, the sample was passivated with a $760 \AA$ A thick $\mathrm{Si}_{x} \mathrm{~N}_{y}$ layer.

Devices with $\mathrm{T}$-shaped layout, $0.7 \mu \mathrm{m}$ nominal gate length, and $150 \mu \mathrm{m}$ gate width were fabricated. Gate-source and gatedrain spacings were $0.7 \mu \mathrm{m}$ and $2.0 \mu \mathrm{m}$, respectively. Transfer length method (TLM) patterns were defined on the n-layer with contacts spacing of $2,3,4,5$, and $7 \mu \mathrm{m}$.

\section{Measurements And Results}

Resistance between different TLM contacts was measured at $300 \mathrm{~K}$ using a four-point probe arrangement. The sheet resistance $\left(R_{s h}\right)$ of the n-layer was monitored by TLM measurements at different etch depth of the p-layer, as shown in Fig. 2. $R_{s h}$ decreased from about $3800 \Omega /$ sq for unetched devices to about $2000 \Omega / \mathrm{sq}$ for the $600 \AA$ A etch depth. Such a decrease was associated with the removal of the p-layer and consequent reduction of the n-layer depletion region from 600 to $340 \AA$, assuming nominal doping concentrations. Depletion thicknesses were estimated by one-dimensional (1-D) Poisson simulations, as shown in Fig. 1. The theoretical minimum for $R_{s h}$ is obtained when the p-layer is completely removed, and it is expected to increase for overetches due to $\mathrm{n}$-channel thickness reduction.

Contact resistance was estimated to be lower than $1 \Omega \cdot \mathrm{mm}$ in all cases. A reliable determination of contact resistance was not possible due to the high value of sheet resistance and to geometric tolerances in TLM pattern definition. The coefficient of

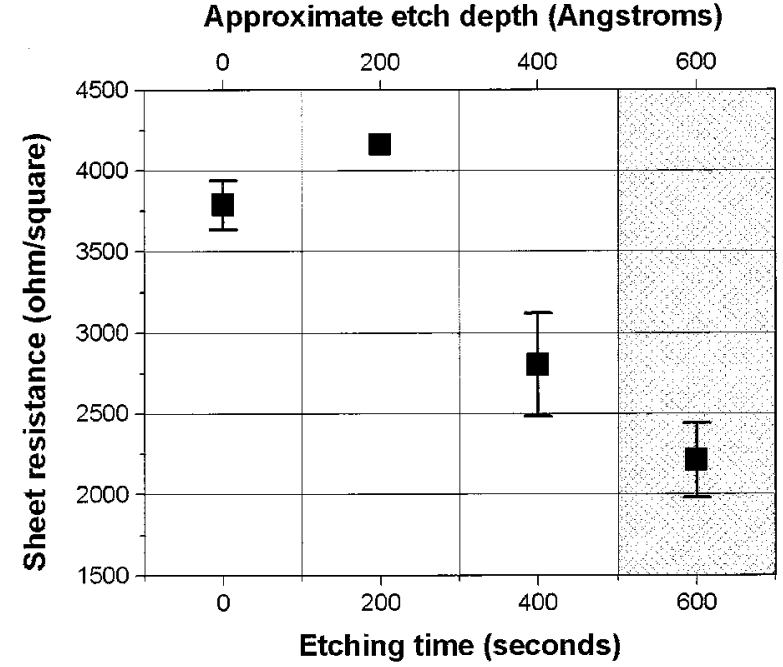

Fig. 2. Measured n-layer sheet resistances as function of p-layer etch depth. Average sheet resistances were $3787,4160,2800,2210 \Omega / \mathrm{sq}$ for unetched, 200, 400 , and $600 \AA$ etch depths, respectively. The shaded area reflects the end of the $\mathrm{p}^{+}$-GaN layer (nominal thickness: $500 \AA$ ). The last etch results in a slight thinning of the n-doped $\mathrm{GaN}$ channel.

determination $R^{2}$ varied between 0.996 and 0.9999 , validating the reliability of sheet resistance data.

Fig. 3 shows dc (solid line) and $80 \mu$ s pulsed (open circles) current-voltage $(I-V)$ characteristics. The pulsed $I-V$ was used to evaluate RF-dispersion. Fig. 3(a) corresponds to unetched conditions, whereas Fig. 3(b) corresponds to complete removal of the p-layer. The unetched device [Fig. 3(a)] yields a maximum saturation current $\left(V_{G S}=2 \mathrm{~V}\right)$ of $0.60 \mathrm{~A} / \mathrm{mm}$, increasing slightly to $0.66 \mathrm{~A} / \mathrm{mm}$ after removal of the p-layer [Fig. 3(b)]. Pinch-off voltage $\left(V_{p}\right)$ was $-12 \mathrm{~V}$ and maximum extrinsic transconductance $\left(g_{m}\right)$ was $60 \mathrm{mS} / \mathrm{mm}$. RF dispersion was negligible before p-layer removal, but significant after full-depletion (400 ̊ etch) or complete removal (600 ̊̊ etch) of the layer, supporting the effectiveness of such a cap layer for suppression of RF-dispersion. This layer screens the n-layer from surface states affecting the n-layer depletion width, as schematically shown in Fig. 1.

Associated with the decrease in sheet resistance there was an increase in small-signal RF performance. $f_{t}$ and $f_{\max }$ improved from 4 to 10.0 and from 4.7 to $10.0 \mathrm{GHz}$, respectively, at a bias point of $V_{D S}=15 \mathrm{~V}$ and $I_{D S}=0.2 \mathrm{~A} / \mathrm{mm}$. The improvement was progressive up the $400 \AA$ etch. Such an increase in frequency performance was related to the decrease in effective gate length due to the removal or complete depletion of the p-layer from the access regions. The p-layer was in fact conductive enough to modulate the access regions next to the gate even at radio frequencies. Thinning and eventual removal of this layer implied a reduction in virtual gate length and a progressive improvement in high frequency performances.

In agreement with previously published data [2]-[4], the deposition of a $\mathrm{Si}_{x} \mathrm{~N}_{y}$ surface passivation layer was found to induce an increase in electron concentration and a decrease in RF-dispersion. The $I-V$ characteristics of a passivated device are shown in Fig. 3(c). The maximum saturation current $\left(V_{G S}=\right.$ $2 \mathrm{~V})$ increased from 0.66 to $0.72 \mathrm{~A} / \mathrm{mm}$, and $f_{t}\left(f_{\max }\right)$ increased from 10 to 13 (14) GHz. 


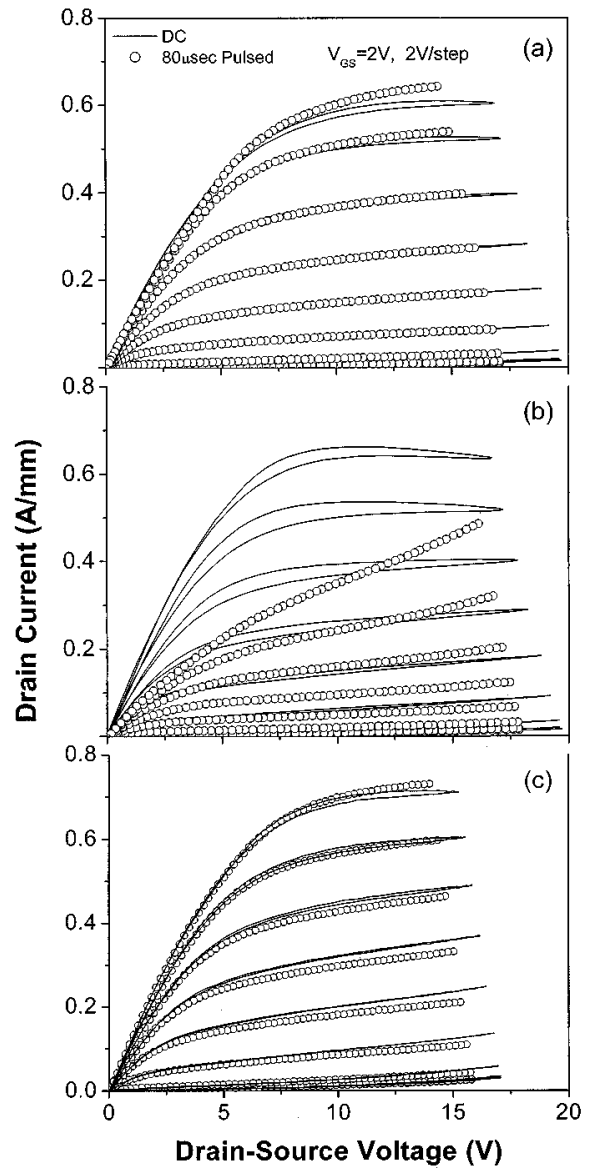

Fig. 3. $I_{D}-V_{D}$ characteristics as function of p-layer thickness. Gate-source voltage varied between 2 and $-16 \mathrm{~V}$. Continuous lines correspond to dc measurements and open-circles refer to $80 \mu$ s pulsed measurements from pinch-off to open channel (simulating AC operation). For the same device, electrical characteristics were taken (a) in unetched conditions, (b) after complete removal of the p-doped overlayer, and (c) after $\mathrm{Si}_{x} \mathrm{~N}_{y}$ passivation.

\section{DisCUSSION AND CONCLUSIONS}

The present study highlights that the presence of a p-type overlayer on GaN FETs could be a viable solution for suppression of RF-dispersion at epilayer design level. High p-doping revealed to be effective in screening the channel from surface state variations, but the magnesium trap-depth was not deep enough to prevent modulation of access regions at radio frequencies. Extended modulation implies a longer effective gate length, with a decrease in $f_{t}$ and $f_{\max }$. Such a modulation could be prevented by increasing the $\mathrm{p}$-layer acceptor depth. Increasing the p-layer acceptor depth would still screen the channel from surface state effects, with no effect on RF performance. Passivation of devices by $\mathrm{Si}_{x} \mathrm{~N}_{y}$ has been successful in RF-dispersion suppression, recovering the characteristics obtained before removing the p-layer from the access regions. The reproducibility of an epilayer compared to a deposited $\mathrm{Si}_{x} \mathrm{~N}_{y}$ thin-film is a highly desirable feature from the processing point of view. Moreover, the better understanding of the surface trap inhibition mechanism in the case of a doped capping layer compared to a silicon nitride overlayer will allow optimization at design level and increase processing latitude.

\section{REFERENCES}

[1] C. Nguyen, N. X. Nguyen, and D. E. Grider, "Drain current compression in GaN MODFETs under large-signal modulation at microwave frequencies," Electron. Lett., vol. 35, pp. 1380-1382, 1999.

[2] B. M. Green, K. K. Chu, E. M. Chumbes, J. A. Smart, J. R. Shealy, and L. F. Eastman, "The effect of surface passivation on the microwave characteristics of undoped AlGaN/GaN HEMTs," IEEE Electron Device Lett., vol. 21, pp. 268-270, June 2000.

[3] S.C. Binari, K. Ikossi, J. A. Roussos, W. Kruppa, D. Park, H. B. Dietrich, D. D. Koleske, A. E. Wickenden, and R. L. Henry, "Trapping effects and microwave power performance in AlGaN/GaN HEMTs," IEEE Trans Electron Devices, vol. 48, pp. 465-471, Feb. 2001.

[4] R. Vetury, N. Q. Zhang, S. Keller, and U. K. Mishra, "The impact of surface states on the dc and RF characteristics of AlGaN/GaN HFETs," IEEE Trans. Electron Devices, vol. 48, pp. 560-566, Mar. 2001.

[5] X. Hu, A. Koudymov, G. Simin, J. Yang, M. Asif Khan, A. Tarakji, M. S. Shur, and R. Gaska, " $\mathrm{Si}_{3} \mathrm{~N}_{4} / \mathrm{AlGaN} / \mathrm{GaN}$-metal-insulator-semiconductor heterostructure field-effect transistors," Appl. Phys. Lett., vol. 79, pp. 2832-2834, 2001.

[6] J. C. Zolper, R. J. Shul, A. G. Baca, R. G. Wilson, S. J. Pearton, and R. A. Stall, "Ion-implanted GaN junction field effect transistor," Appl. Phys. Lett., vol. 68, pp. 2273-2275, 1996.

[7] L.Zhang, L. F. Lester, A. G. Baca, R. J. Shul, P. C. Chang, C. G. Willison, U. K. Mishra, S. P. DenBaars, and J. C. Zolper, "Epitaxially-grown GaN junction field effect transistors," IEEE Trans. Electron Devices, vol. 47, pp. 507-511, Mar. 2000.

[8] B. Heying, I. Smorchkova, C. Poblenz, C. Elsass, P. Fini, S. DenBaars, U. Mishra, and J. Speck, "Optimization of the surface morphologies and electron mobilities in GaN grown by plasma-assisted molecular epitaxy," Appl. Phys. Lett., vol. 77, pp. 2885-2887, 2000. 